\title{
Energy absorption of an egg-box material
}

\author{
V.S. Deshpande, N.A. Fleck* \\ Department of Engineering, Cambridge University, Trumpington Street, Cambridge CB2 1PZ, UK
}

Received 21 February 2002; accepted 3 June 2002

\begin{abstract}
Conical frustra made from leaded gun-metal have been compressed axially. Collapse is either by a travelling plastic hinge or by tearing. An analytical model is developed for the travelling plastic hinge in a rigid, ideally plastic solid; its predictions are compared with the observed response, and with those of an axisymmetric finite element analysis. The travelling hinge mechanism is also observed in the compressive collapse of an egg-box material comprising a square array of conical frustra. Collapse mechanism maps are constructed for the egg-box material, and they show the regimes of dominance of elastic buckling, material tearing and the travelling plastic hinge. The maps are useful for selecting egg-box geometries that maximise the energy absorption per unit mass at any prescribed value of collapse stress. The optimisation indicates that the egg-box material has a similar energy absorption capacity to that of hexagonal honeycombs and is superior to that of metal foams.
\end{abstract}

(C) 2002 Elsevier Science Ltd. All rights reserved.

Keywords: Energy absorption; Tearing; Travelling plastic hinge; Elastic buckling; Compressive strength; Conical frustum

\section{Introduction}

In recent years a number of new micro-architectured materials have been developed for the multi-functional design of lightweight structures. Lattice materials are periodic truss structures with a sufficiently large connectivity at each node to provide for structural rigidity: under all applied loadings the individual struts deform by stretching rather than by bending (Deshpande and Fleck, 2001; Deshpande et al., 2001; Wicks and Hutchinson, 2001). Consequently, the stiffness and strength of lattice materials scale linearly with their relative density $\bar{\rho}$.

\footnotetext{
* Corresponding author. Fax: +44-1223-332662.

E-mail address: naf1@eng.cam.ac.uk (N.A. Fleck).
} 
(a)
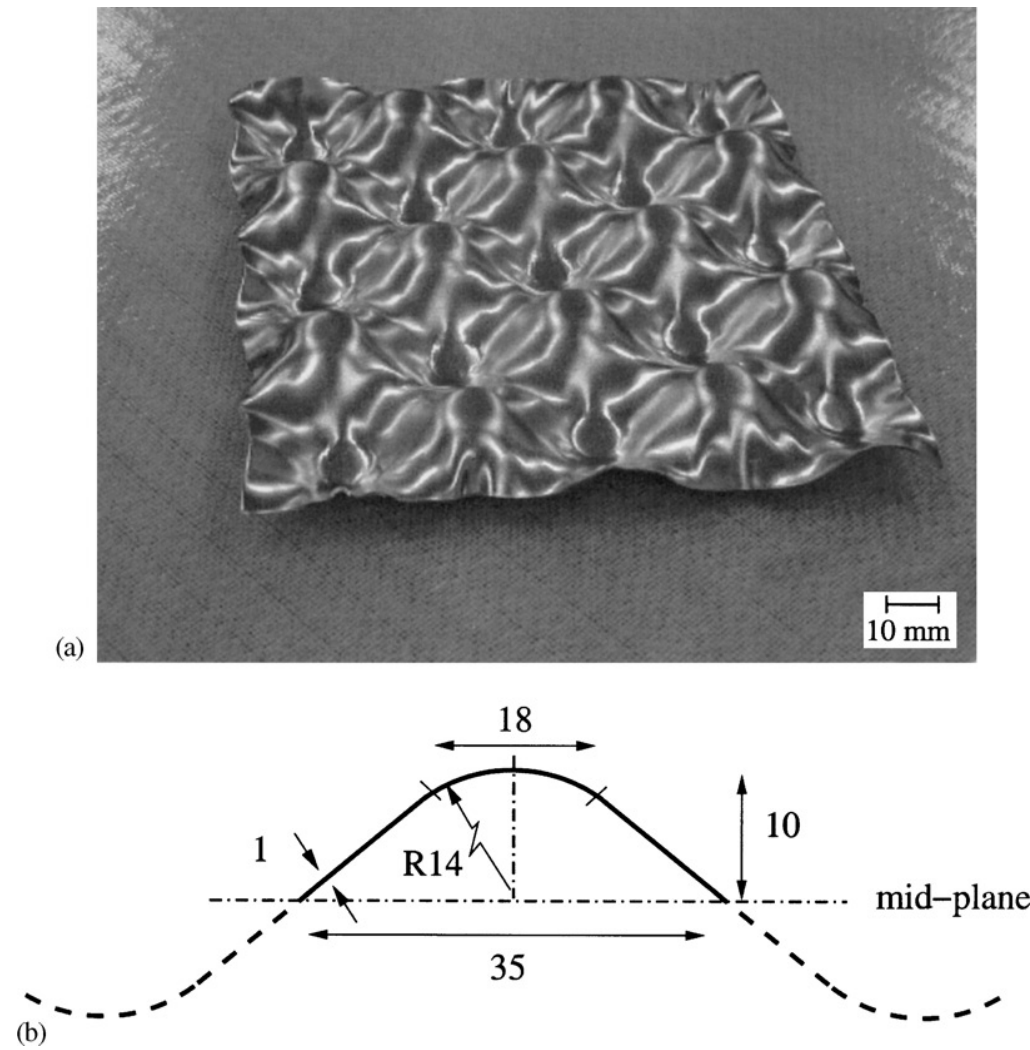

Fig. 1. (a) Photograph of the egg-box material made by cold-pressing commercially pure aluminium. (b) Sketch of the cross-section of the egg-box material, from crown to adjacent trough. All dimensions are in mm. The solid line represents a unit cell of the egg-box material, and is a conical frustum with a hemispherical crown.

Lattice materials collapse in compression by the elastic buckling of struts at $\bar{\rho}<1 \%$ and by plastic yield at higher values of $\bar{\rho}$. Provided $\bar{\rho}<20 \%$ the struts are sufficiently slender for the compressive collapse load to drop steeply in magnitude beyond the initial peak value: this feature is not desirable in energy absorption. For relative densities higher than $20 \%$, the struts are stocky and collapse at an almost constant load, making for high energy absorption. For many energy absorption applications a material of low density is required and lattice materials are not the best choice. Instead, hexagonal honeycombs, metal foams and egg-box materials are candidates; the geometry of the egg-box material used in the present study is shown in Fig. 1. Metal honeycombs are currently used for energy absorption within the cores of sandwich panels, while metal foams can be manufactured to complex near nett shape by powder metallurgical methods (Banhart, 2000). Egg-box material is potentially cheaper than lattice material, honeycomb or metal foam as it can be cold or hot stamped from a wrought sheet in a single step operation. 
Hexagonal honeycombs give high energy absorption by the formation of a succession of folds of each cell, with hoop stretching of the cell wall between each fold. The formation of each successive fold leads to large oscillations in the collapse stress. In contrast, open-cell metal foams and the current generation of commercially produced closed-cell metal foams absorb energy primarily by the bending of the cell edges at stationary plastic hinges. Again, fluctuations in the macroscopic stress versus strain curve are observed, but now the cause is the successive collapse of layers of the foam. In the current study, we investigate experimentally and theoretically the compressive collapse of egg-box material in order to determine its ability to absorb energy. We shall show that the primary mode of collapse is by a travelling plastic hinge within each periodic cell of the egg-box. The same collapse mode is exhibited by a closed-top conical frustum, and so the study begins with an investigation of the axial compressive response of a closed-top conical frustum.

\section{Compressive behaviour of conical frustra}

A number of researchers have investigated experimentally and theoretically the compressive collapse of open-top conical frustra, see for example Mamalis et al. (1986a, b). Collapse is by a variety of plastic folding mechanisms (axisymmetric or diamond patterns) which resemble the collapse mechanisms of thin walled cylindrical shells. Alghamdi (2001) has recently reviewed these and related investigations. In this section we investigate the compressive deformation behaviour of conical frustra with closed tops. Then in the following sections, the results for conical frustra are used to interpret the transverse compressive collapse of egg-box material.

\subsection{Experimental investigation}

Conical frustra of geometry sketched in Fig. 2a, were machined from solid circular bars of leaded gun-metal of wt. $\% \mathrm{Cu}-7 \mathrm{~Pb}-7 \mathrm{Sn}-3 \mathrm{Zn}$. Gun-metal was chosen as it has a high tensile ductility of about $22 \%$ : tearing of the material is a potential failure mechanism as will be seen later in the paper. All specimens had a wall thickness $t=1 \mathrm{~mm}$ and base diameter $D=35 \mathrm{~mm}$. The transition in shape between the flat top of radius $a$ and the conical portion of the frustum was sharp, with a radius of curvature $c$ on the order of microns. Four conical frustra, with $a=2$ and $5 \mathrm{~mm}$ and cone angles $\omega=30^{\circ}$ and $50^{\circ}$, were manufactured and tested; the cone angle $\omega$ is defined in Fig. 2a.

Each conical frustum was compressed between parallel steel platens on a screw-driven test machine. All tests were performed at a nominal macroscopic strain rate of $10^{-3} \mathrm{~s}^{-1}$. The applied load $P$ and the relative displacement $u$ of the platens were recorded and plotted in Fig. 2b. Two competing deformation modes were observed:

1. The specimen with a large cone angle $\omega=50^{\circ}$ and a small top $a=2.5 \mathrm{~mm}$ tore as it underwent plastic straining within the knuckle. After tearing, the specimen 
(a)
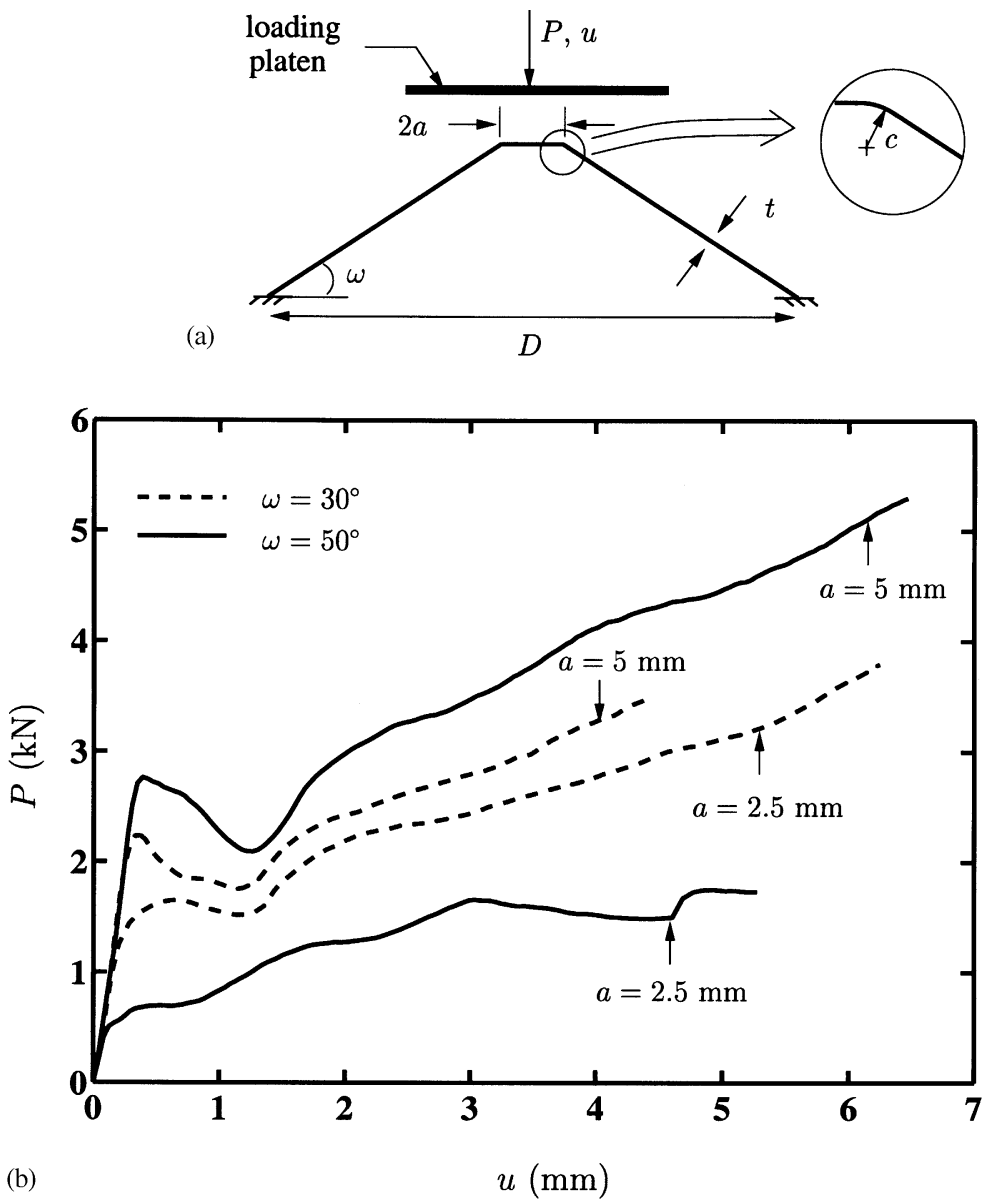

Fig. 2. (a) Sketch of conical frustum and the loading arrangement. (b) Measured load versus displacement curves for conical frustra made from leaded gun-metal.

deformed by the in-extensional plastic folding mechanism for open-top conical shells as reported by Mamalis et al. (1986b).

2. A travelling plastic hinge was observed in the remaining geometries. The characteristics of this travelling hinge are clear from the diametrical section of the specimen with geometry $\omega=30^{\circ}, a=2.5 \mathrm{~mm}$ and axial displacement $u=6 \mathrm{~mm}$, as shown in Fig. 3. A circumferential knuckle forms the travelling plastic hinge and leads to the progressive inversion of the conical frustum from its apex.

The three conical frustra that deformed by the travelling hinge mechanism showed an initial load peak associated with the initiation of the travelling hinge. The load dropped over the ensuing $1 \mathrm{~mm}$ of axial displacement followed by an increase in load as the hinge propagated. On the other hand, the conical frustum that underwent 


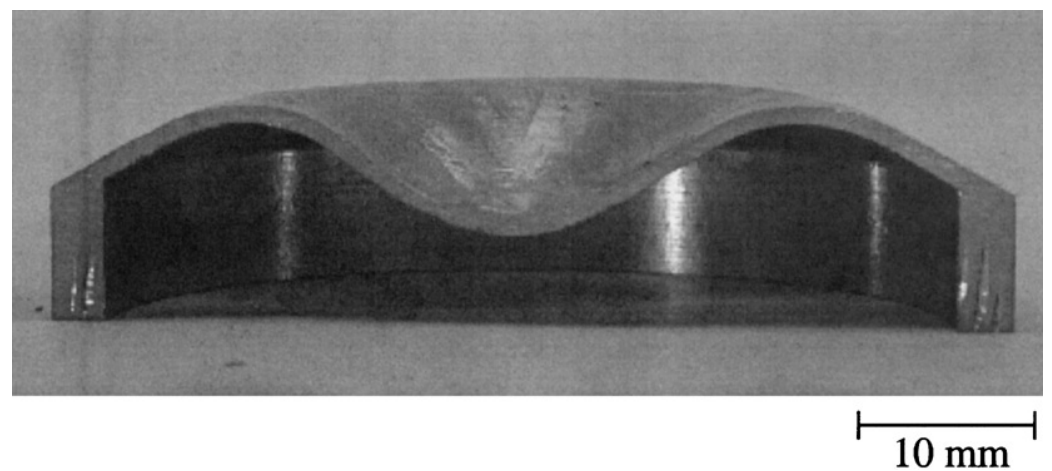

Fig. 3. Photograph of the $\omega=30^{\circ}(a=2.5 \mathrm{~mm})$ conical frustum deformed to $u \approx 6 \mathrm{~mm}$ and section along its mid-plane. The knuckle which forms the travelling plastic hinge is evident.

tearing displayed no load peak and the load versus displacement curve was much flatter.

\subsection{Travelling hinge model}

The experiments discussed above indicate that closed-top conical frustra deform by the travelling hinge mode of deformation provided material tearing is avoided. Here we modify the approximate analysis of Calladine (1986) for the inversion of a spherical shell under a point central load to the case of a conical frustum compressed between two frictionless rigid platens. The conical frustum is made from a rigid, ideally plastic solid of yield strength $Y$, and uniform wall thickness $t$.

Consider the inversion of a conical frustum by the outward propagation of a narrow toroidal knuckle as shown in Fig. 4a. A frictionless loading platen applies a ring load to the knuckle top and active plastic straining occurs only within the knuckle region. An analytical solution may be devised upon adopting a simplified yield surface for the conical shell. Active plastic straining within the knuckle is by a combination of hoop stretching and longitudinal bending. We assume that yield of an axisymmetric shell element is described by the resultant hoop stress $N_{\phi}$ and the longitudinal bending moment $M_{\mathrm{L}}$. For simplicity we adopt the circumscribing yield locus (Onat, 1955),

$$
\begin{aligned}
& \left|N_{\phi}\right|=N_{\mathrm{o}} \equiv Y t, \\
& \left|M_{\mathrm{L}}\right|=M_{\mathrm{o}} \equiv \frac{Y t^{2}}{4},
\end{aligned}
$$

where $N_{\mathrm{o}}$ and $M_{\mathrm{o}}$ are the plastic values of the stress resultant and the bending moment, respectively. At yield one or both of these relations is satisfied.

\subsection{The stress state within the knuckle and central inverted frustum}

The knuckle region is connected to the inverted and conical regions by two travelling circumferential plastic hinge circles of radii $r \pm l / 2$ as sketched in Fig. 4a. In a frame 

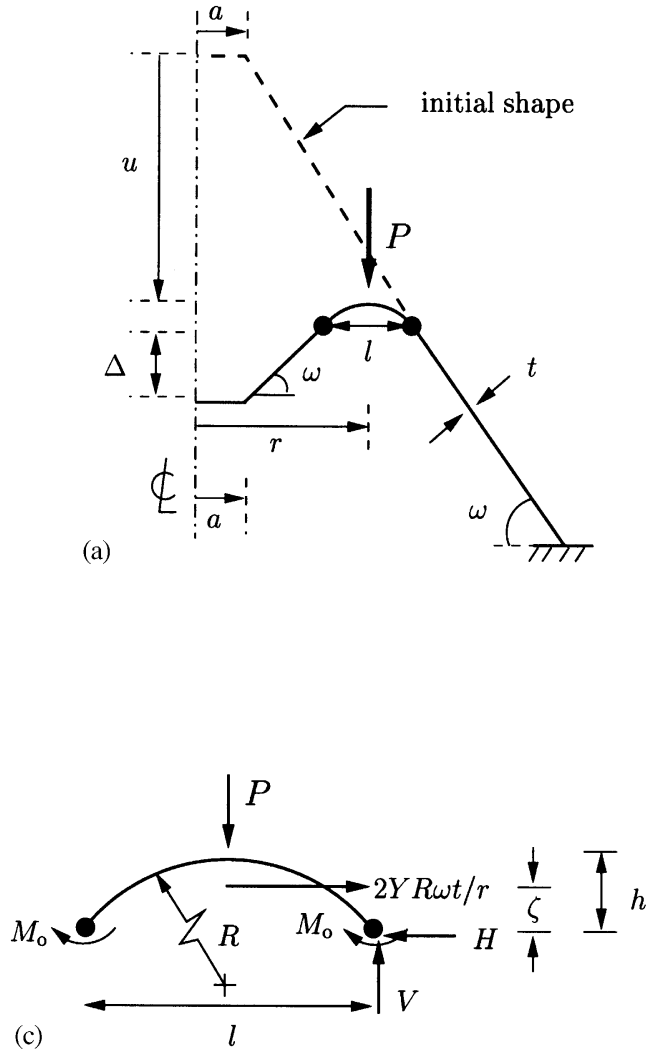
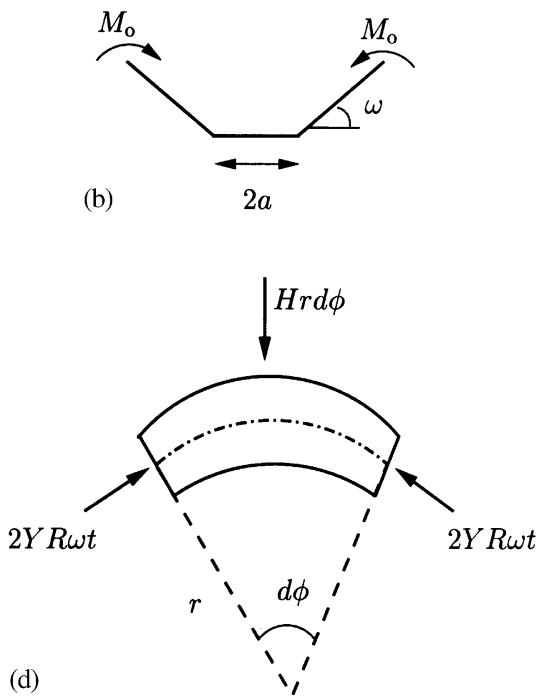

Fig. 4. The travelling hinge model. (a) sketch of the cross-section of the partially inverted frustum, (b) free body diagram showing the cross-section of the inverted central region, (c) free body diagram of the knuckle with a unit circumference at radius $r$, and (d) free body diagram showing the plan view of a circumferential segment of the knuckle subtending a polar angle $\mathrm{d} \phi$ at the centre.

of reference which moves with the toroidal knuckle, material enters the knuckle at the outer hinge and a longitudinal curvature is imposed; when material elements leave the knuckle by the inner hinge, this curvature is removed. Axisymmetric shell elements within the knuckle are subjected to a longitudinal bending moment $M_{\mathrm{L}}$ that decreases from $+M_{\mathrm{o}}$ at the outer plastic hinge to $-M_{\mathrm{o}}$ at the inner hinge, while the hoop bending moment vanishes throughout the knuckle. Since the equations of equilibrium require $M_{\mathrm{L}}$ to vary continuously, we argue that $\left|M_{\mathrm{L}}\right|<M_{\mathrm{o}}$ within the knuckle and so no change in longitudinal curvature occurs between the two hinge circles. The toroidal knuckle rotates instantaneously about the outer hinge, and in order for the inverted conical frustum to be rigid, the entry and exit hinge-circles lie on the same horizontal plane as indicated in Fig. 4a. During an incremental rotation of the knuckle about the outer hinge each elemental hoop within the knuckle displaces with an inward radial component. Thus, the knuckle undergoes compressive hoop straining and it follows 
from the circumscribing yield locus that the hoop stress within the entire knuckle region attains the compressive yield strength.

Fig. $4 \mathrm{~b}$ shows the stress resultants which act on the central inverted frustum. The outer edge of inverted frustum is subjected to a longitudinal bending moment $M_{\mathrm{L}}=-M_{\mathrm{o}}$. Vertical equilibrium of the inverted frustum dictates that the vertical component of the stress resultant on the outer edge of the inverted frustum equals zero. Also, the longitudinal bending moment is a maximum on the outer edge since the outer edge is at a moving plastic hinge. Consequently, the shear force vanishes and no stress resultants act on the outer edge of the inverted central frustum.

\subsection{Equilibrium relations}

The cross-section of the knuckle and the stress resultants that act on a segment of unit circumference at radius $r$ are sketched in Fig. 4c. The ends of the knuckle have a slope of $\pm \omega$, and the profile of the knuckle is a circular arc of radius $R$. Geometrical considerations dictate that the height $h$ of the knuckle and the elevation $\zeta$ of the centroid above the plane of the hinge circles are given by

$$
h=R(1-\cos \omega)
$$

and

$$
\zeta=R\left(\frac{\sin \omega}{\omega}-\cos \omega\right) .
$$

Upon assuming that $l \ll r$, equilibrium in the axial (vertical) direction gives

$$
V=\frac{P}{2 \pi r},
$$

where $P$ is the total compressive force exerted by the rigid plates and $V$ is the vertical force per unit length at the outer hinge circle. At the outer hinge circle the force resultant is tangential to the longitudinal direction since $M_{\mathrm{L}}$ is a maximum at the hinge circle and thus it follows that the inner radial force per unit length $H$ is

$$
H=\frac{P}{2 \pi r \tan \omega} \text {. }
$$

Now consider radial equilibrium of a segment of the knuckle subtending a small polar angle $\mathrm{d} \phi$ as shown in Fig. $4 \mathrm{~d}$. The inward radial force balances the outward radial force due to compressive hoop yield within the knuckle, to give

$$
H r \mathrm{~d} \phi=2 Y t R \omega \mathrm{d} \phi .
$$

Elimination of $H$ from Eqs. (3b) and (4) gives

$$
P=4 \pi Y t R \omega \tan \omega \text {. }
$$

In order to determine the knuckle radius $R$ we consider moment equilibrium about the hoop direction. Recall that the stress resultants on a segment of the knuckle with unit circumference at radius $r$ are sketched in Fig. 4c. Then on taking moments about the 
outer hinge, we obtain

$$
\frac{P l}{4 \pi r}=2 M_{\mathrm{o}}+\frac{2 Y t \omega R^{2}}{r}\left(\frac{\sin \omega}{\omega}-\cos \omega\right)
$$

and an explicit expression for $R$ follows via (5) and the relation $l=2 R \sin \omega$,

$$
R=\left[\frac{M_{\mathrm{o}} r}{Y t(2 \omega-\sin 2 \omega)}\right]^{1 / 2} .
$$

An expression for the compressive force $P$ is obtained in terms of the current radius $r$ by combining Eqs. (5) and (7) to obtain

$$
P=\frac{2 \pi \omega \tan \omega}{(2 \omega-\sin 2 \omega)^{1 / 2}} Y t^{3 / 2} r^{1 / 2} .
$$

For thin walled shells, it is reasonable to assume that the knuckle dimensions are much smaller than the axial displacement $u$ of the rigid platen. Then the nominal compressive displacement $u$ of the conical frustra is related to $r$ through

$$
r \approx a+u \cot \omega
$$

and the load versus displacement relation of the conical shell can be expressed as

$$
\frac{P}{Y t^{2}}=\frac{2 \pi \omega \tan \omega}{(2 \omega-\sin 2 \omega)^{1 / 2}}\left[\frac{a}{t}+\frac{u}{t} \cot \omega\right]^{1 / 2} .
$$

In some of the experiments described above, tearing was observed within the incipient knuckle. The longitudinal bending strain $\varepsilon_{\mathrm{L}}$ induced by the travelling knuckle is given by

$$
\varepsilon_{\mathrm{L}}=\frac{t}{2 R}=\left[\frac{2 \omega-\sin 2 \omega}{a / t+u / t \cot \omega}\right]^{1 / 2} .
$$

It is recalled that tearing occurred in the conical frustum of geometry $\omega=50^{\circ}$ and $a / t=2.5$ made from gun-metal of ductility $22 \%$. The maximum bending strain $\varepsilon_{\mathrm{L}}$ is $55 \%$ by (11), and so tearing is anticipated.

\subsubsection{Comparison of the model with finite element calculations and with measurements}

The accuracy of the analytical model is gauged in two steps. First, it is compared with finite element (FE) calculations for an elastic, ideally plastic solid and second it is compared with the measured responses of the conical frustra made from gun-metal, as summarised in Fig. 2.

The FE calculations were performed using the general purpose finite element package ABAQUS (HKS, 1997) with the conical frustra modelled by about 320 axisymmetric quadratic shell elements (SAX2 element of ABAQUS). The base of the frustra was constrained to have no lateral displacements and no rotation. Loading was applied through prescribed displacements of rigid loading platens, with the contact between the conical frustum and the rigid surfaces modelled by a frictionless contact surface as 
provided by ABAQUS. J2 flow theory was employed and the solid was taken to be elastic - perfectly plastic with a yield strain $\varepsilon_{Y}=0.1 \%$ and a Poisson's ratio $v=0.3$.

A comparison between the load versus displacement curves from the FE calculations and the analytical travelling hinge model is shown in Fig. 5a for cones $(a / t=0)$ and in Fig. $5 \mathrm{~b}$ for conical frustra $(a / t=5)$. For the cones, the travelling hinge solution is in good agreement with the FE calculations once a travelling hinge has become established at $u / t>0.2$. For the conical frustra both perfect and imperfect finite element geometries are considered as follows:

1. Perfect conical frustra, with a sharp transition between the flat top and the conical shell and labelled as $c=0$ in Fig. 5b. The FE calculations show an initial peak in the load versus displacement response corresponding to initiation of the travelling hinge. After the travelling hinge has become established, the analytical travelling hinge solution agrees well with the FE calculations.

2. "Imperfect" frustra, with a local radius at the transition given by $c / t=1.7$. The FE calculations shown in Fig. $5 \mathrm{~b}$ reveal that the initial load peak is now absent and adequate agreement is noted between the FE and rigid-plastic analytical calculations for $u / t>0.5$.

It is concluded that the travelling hinge model, despite its simplicity, gives an accurate description of the collapse of conical frustra made from an elastic perfectly plastic solid.

The second step in gauging the accuracy of the travelling hinge model is to compare its predictions with the observed collapse response of the gun-metal frustra. To achieve this we measured the tensile stress versus strain behaviour of the leaded gun-metal, see Fig. 6a. The gun-metal has a yield strength of $170 \mathrm{MPa}$, an ultimate tensile strength of $284 \mathrm{MPa}$ and a tensile ductility of $22 \%$. Comparisons between the measured load versus displacement response of the conical frustra and the analytical travelling hinge model are given in Fig. 6b for an assumed flow strength $Y=(170+284) / 2=227 \mathrm{MPa}$. The comparisons are made only for the case $a=5 \mathrm{~mm}$ and $\omega=30^{\circ}$ and $50^{\circ}$ as these frustra did not tear. Reasonable agreement between the model predictions and the experimental measurements is seen in light of the fact that the analytical model neglects the effects of strain hardening.

\section{Compressive behaviour of the egg-box material}

Commercially pure (CP) aluminium sheets of thickness $1.0 \mathrm{~mm}$ were cold pressed ${ }^{1}$ into an egg-box shape with overall dimensions $170 \times 170 \times 20 \mathrm{~mm}^{3}$ as shown in Fig. 1a. The egg-box geometry can be approximated by a square array of conical frustra with each frustum of half the height of the egg-box material; the base diameter of the frustum is taken as the spacing between adjacent crowns of the egg-box. For

\footnotetext{
${ }^{1}$ Cellbond Composites Ltd., 5 Stukeley Business Centre, Blackstone Road, Huntingdon PE29 6EF, UK (Ashmead, 2000).
} 

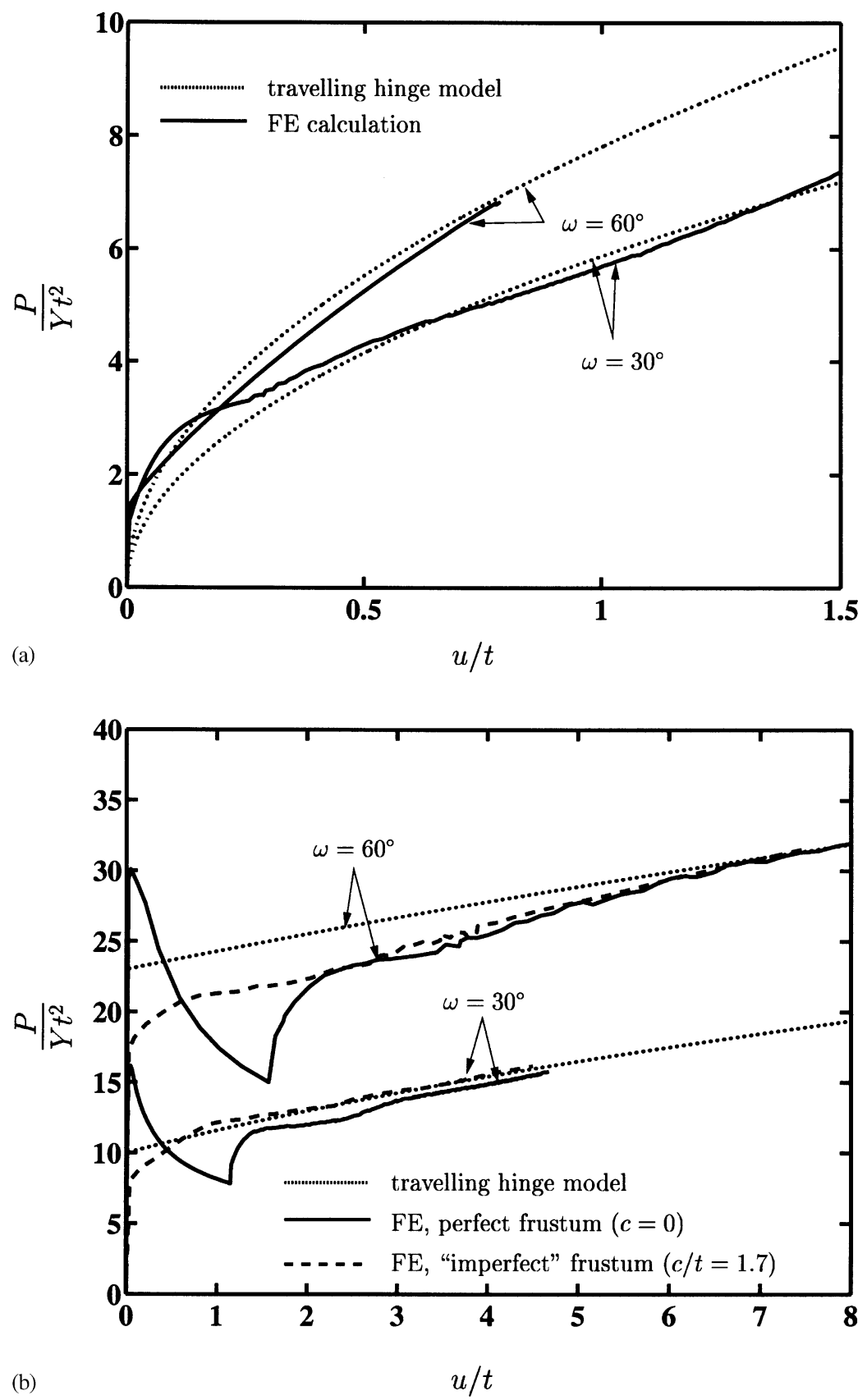

Fig. 5. Comparison between the FE and travelling hinge model predictions of the load versus displacement response for: (a) cones with $a / t=0$, (b) conical frustra with $a / t=5$. In the FE calculations, the yield strain $\varepsilon_{Y}$ was taken as $0.1 \%$. 


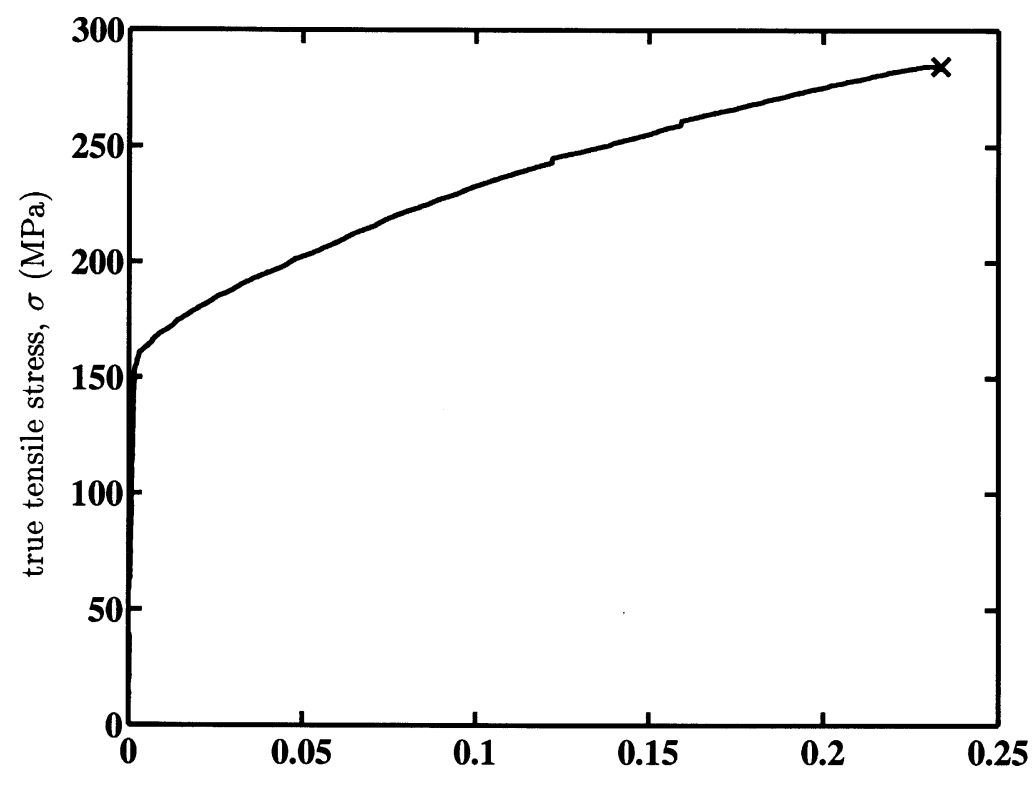

(a)

logarithmic tensile strain, $\epsilon$

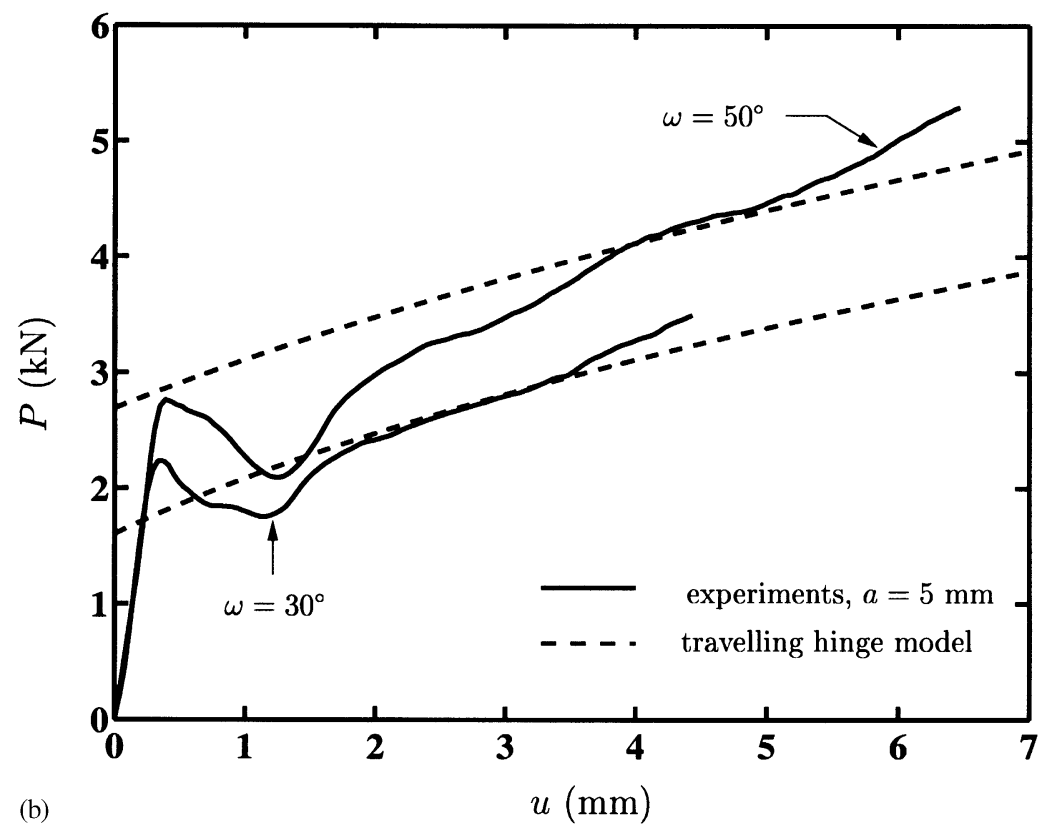

Fig. 6. (a) Measured tensile stress versus strain curve of the leaded gun-metal. (b) Comparison between the measured load versus displacement response and the predictions of the rigid, perfectly plastic travelling hinge model $(Y=227 \mathrm{MPa})$ for conical frustra with $a=5 \mathrm{~mm}$. 
the manufactured egg-box, each frustum had a height of $10 \mathrm{~mm}$ and top cap and base diameters of 18 and $35 \mathrm{~mm}$, respectively. The crowns of the egg-box were not flat: they comprised hemispherical shells of radius $R=14 \mathrm{~mm}$, as sketched in Fig. 1b.

Prior to testing, the egg-box specimen was annealed for $4 \mathrm{~h}$ at $300^{\circ} \mathrm{C}$ in an air furnace to reduce the effects of the work-hardening of the solid material due to the cold-pressing. The compressive response of the egg-box specimen was measured with full in-plane constraint: in-plane lateral expansion of the egg-box material was prevented by placing the specimen within a steel box with inner dimensions identical to the projected plan dimensions of the egg-box specimen. The loading platens were covered with PTFE tape to reduce friction, and the compression test was performed using a screw driven test machine at a nominal strain rate of $10^{-3} \mathrm{~s}^{-1}$. The applied load was measured by the load cell on the test machine and was used to define the nominal compressive stress on the specimen; the nominal axial strain was measured via a clip gauge fixed between the loading platens.

The measured compressive stress versus strain response of the egg-box material is shown in Fig. 7a. The stress versus strain curve comprises two regimes of behaviour: first, the stress increases gently with strain up to the densification strain, and second it rises steeply. A visual examination of the deformed specimen revealed that the deformation mode in the first regime involved the progressive inversion of the conical frustra, with the load transmitted from the rigid platens to the egg-box through circumferential knuckles. This closely resembles the travelling hinge mechanism discussed above.

In order to compare the measured response with FE calculations we measured the uniaxial tensile response of the annealed CP aluminium sheet from which the egg-box material was cold-pressed; this curve is plotted in Fig. $7 \mathrm{~b}$. It was found that the $\mathrm{CP}$ aluminium had a yield strength $Y \approx 40 \mathrm{MPa}$, an ultimate tensile strength of about $85 \mathrm{MPa}$ and a tensile ductility of $20 \%$. Axisymmetric finite element calculations were performed on the axial collapse of the unit cell shown in Fig. 1b. Details on the mesh, boundary conditions and the contact problem are the same as described above for the conical frustum. The solid was treated as an isotropic hardening $J 2$ flow theory solid, with the measured uniaxial tensile stress versus strain curve as reported in Fig. $7 \mathrm{~b}$ and a Poisson's ratio $v=0.3$. The calculated stress versus strain response of the egg-box material is plotted as a dashed line in Fig. $7 \mathrm{~b}$ and agrees very well with the measured response of the egg-box material over the entire range of deformation. The FE analysis also revealed that the mode of deformation resembled a travelling hinge mechanism.

\subsection{Analysis of compressive deformation}

It is of practical importance to develop analytical expressions for the collapse strength and energy absorption capacity of the egg-box material. We proceed by making use of the travelling hinge model for the conical frustum. Consider an egg-box material of depth $d$ made from a sheet of thickness $t$. The egg-box comprises a $2 \mathrm{D}$ square array of conical frustra, each with a top radius $a$ and a cone angle $\omega$, see Fig. $1 \mathrm{~b}$. The base of the unit cell for each frustum is assumed to be a square of side $(2 a+d \cot \omega)$; thereby the unit cells fill space. The relative density $\bar{\rho}$ of the egg-box material (ratio 


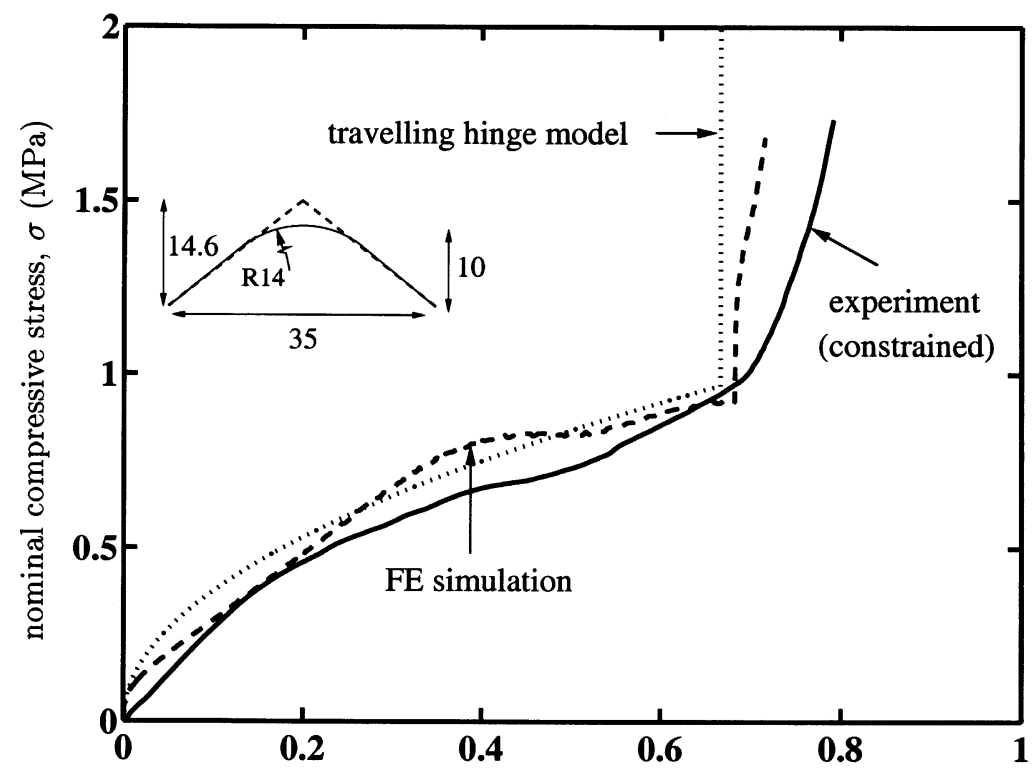

(a)

nominal compressive strain, $\epsilon$

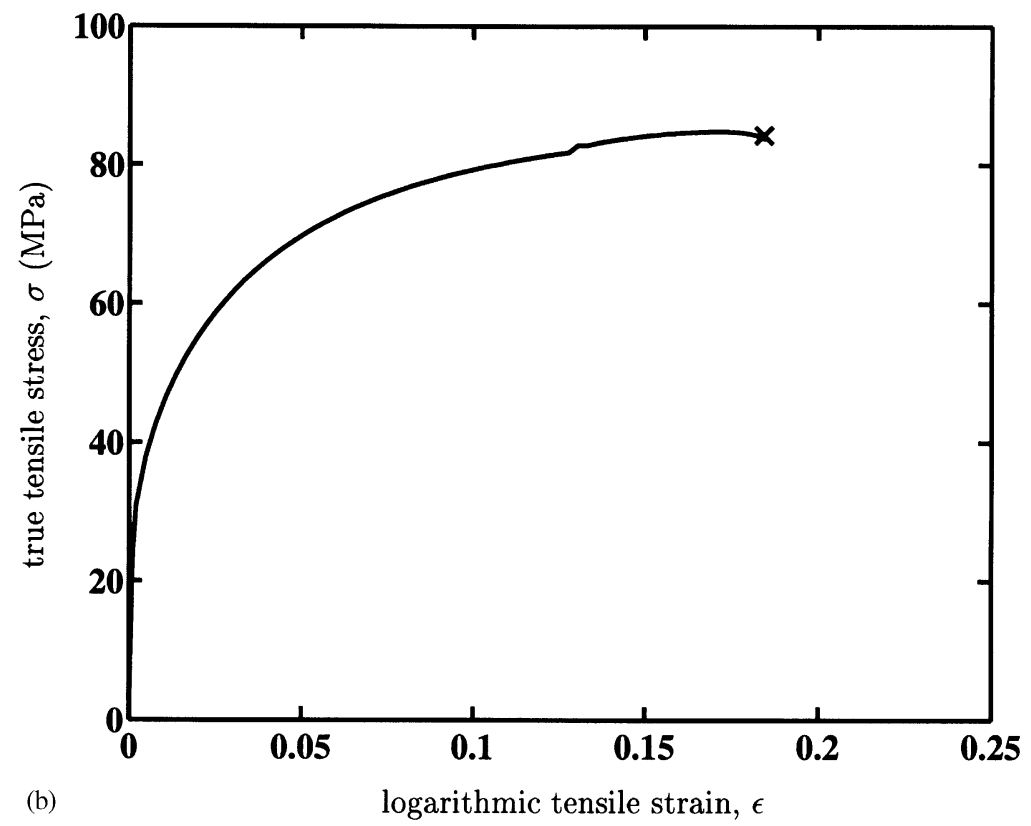

Fig. 7. (a) Comparison between measured, travelling hinge model and FE predictions of the constrained compressive stress versus strain response of the egg-box material. In the insert all dimensions are in $\mathrm{mm}$. (b) Measured tensile stress versus strain curve of the as-received commercially pure (CP) aluminium sheet. 
of the density of the egg-box material to the density of the solid material from which it is made) is given by

$$
\bar{\rho}=\frac{\pi}{4} \bar{t}\left[\frac{\cot \omega+4 \bar{a} \bar{t}+4 \bar{a}^{2} \bar{t}^{2} \sin \omega}{\sin \omega(2 \bar{a} \bar{t}+\cot \omega)^{2}}\right],
$$

where $\bar{a}=a / t$ and $\bar{t}=t / d$. Experimental measurements and FE simulations both show that the mode of deformation of the egg-box material under constrained compression resembles the travelling hinge mode discussed above. Thus, we proceed by employing the travelling hinge model to estimate the compressive stress versus strain response of the egg-box material.

\subsubsection{Travelling hinge deformation mode}

We analyse the deformation of the egg-box material with the unit cell described above and made from a rigid, perfectly plastic material of yield strength $Y$. Each unit cell contains a conical frustum, and the collapse stress $\sigma$ of the egg-box material is related to the axial load $P$ by

$$
\sigma=\frac{P}{(2 a+d \cot \omega)^{2}} \text {. }
$$

Upon substituting for $P$ from (10), the stress versus strain response of the egg-box material is

$$
\frac{\sigma}{Y}=k\left(\bar{a}+\frac{\varepsilon}{2 \bar{t}} \cot \omega\right)^{1 / 2},
$$

where $\varepsilon$ is the nominal axial strain and $k$ a geometrical constant defined by

$$
k=\frac{\pi \omega \tan \omega}{2} \frac{1}{(2 \omega-\sin 2 \omega)^{1 / 2}} \frac{1}{(\bar{a}+\cot \omega / 2 t)^{2}} .
$$

We proceed by defining the densification strain $\varepsilon_{D}$ as the strain at which the inverted portion of the conical frustum makes first contact with the lower rigid platen. Referring to Fig. $4 \mathrm{a}$, the depth $\Delta$ of the inverted region of the conical frustum below the plane of the hinge circles is given by

$$
\Delta=\left(r-\frac{l}{2}-a\right) \tan \omega .
$$

At densification we have

$$
R(1-\cos \omega)+\Delta+2 u=d,
$$

where $2 u$ is the relative displacement of the platens. Combining Eqs. (16) with (15), and noting that $l=2 R \sin \omega$ gives

$$
R\left(1-\cos \omega-\frac{\sin ^{2} \omega}{\cos \omega}\right)+(r-a) \tan \omega+2 u=d .
$$

With our assumption that the knuckle dimensions are much less than the magnitude of the displacement $u$, we can neglect the first term in the above equation. Upon 
substituting for $r$ from Eq. (9) we obtain

$$
\varepsilon_{\mathrm{D}}=\frac{2}{3} \text {. }
$$

This formula is not expected to be accurate for $\omega \ll 1$ since the assumption $R \ll u$ breaks down.

A comparison between the travelling hinge model predictions and the measured stress versus strain response of the egg-box material is shown in Fig. 7a. It is recalled that the actual geometry of the egg-box consists of a conical shell with a shallow hemispherical crown rather than a flat top. For comparison with the analytical model the egg-box geometry is approximated by a conical frustum with $a=0$ and $\omega=40^{\circ}$, as shown by the dashed line in the insert of Fig. 7a. The predicted stress versus strain response of the conical frustum is plotted in Fig. 7a for a yield strength $Y=60 \mathrm{MPa}$. It is truncated at the estimated densification strain $\varepsilon_{\mathrm{D}}$ as stated by Eq. (18) and a vertical line indicates the sharply rising stress beyond the densification strain. We conclude that the travelling hinge model and the predicted densification strain are in good agreement with the measured response.

\subsubsection{Estimate of energy absorption}

Energy absorbers for packaging and crash protection are chosen so that they can absorb energy at a stress level below a prescribed limit. We define the energy absorption per unit volume $W_{\mathrm{v}}$ as the area under the predicted nominal stress versus nominal strain curve for the egg-box material up to the densification strain $\varepsilon_{\mathrm{D}}$,

$$
W_{\mathrm{v}}=\int_{0}^{\varepsilon_{\mathrm{D}}} \sigma \mathrm{d} \varepsilon
$$

Upon substituting (14a) into the above expression, we obtain

$$
\frac{W_{\mathrm{v}}}{Y}=\frac{4}{3} k \bar{t} \tan \omega\left[\left(\bar{a}+\frac{\varepsilon_{\mathrm{D}}}{2 \bar{t}} \cot \omega\right)^{3 / 2}-\bar{a}^{3 / 2}\right] \text {, }
$$

where $k$ has already been defined by Eq. (14b). This is the energy absorbed per unit volume with a maximum stress $\sigma_{\mathrm{D}}$ set by the stress at the densification strain $\varepsilon_{\mathrm{D}}\left(\sigma_{\mathrm{D}}\right.$ is given explicitly by Eq. (14a) with $\varepsilon=\varepsilon_{\mathrm{D}}=2 / 3$ ). The corresponding energy absorption per unit mass is

$$
W_{\mathrm{m}}=\frac{W_{\mathrm{v}}}{\bar{\rho} \rho_{\mathrm{s}}},
$$

where $\rho_{\mathrm{s}}$ is the density of the sheet from which the egg-box material is made.

\subsection{Collapse mechanism maps and energy absorption}

The collapse of egg-box material under constrained compression is by (a) a travelling plastic hinge mechanism, (b) elastic buckling or (c) tearing. In order to determine how the failure mode depends upon the geometry of the egg-box material we require an estimate of the elastic buckling stress for the egg-box material. 


\subsubsection{Elastic buckling of the egg-box material}

The egg-box material will collapse by elastic buckling when the elastic buckling load is less than the load for a travelling plastic hinge. Recall that the elastic buckling load $P_{\mathrm{c}}$ of a perfect conical shell is given by (Seide, 1956)

$$
P_{\mathrm{c}}=\frac{2 \pi E t^{2} \sin ^{2} \omega}{\sqrt{3\left(1-v^{2}\right)}} .
$$

For the case of a conical frustum, Seide (1956) modified this formula to the form

$$
P_{\text {act }}=C P_{\mathrm{c}},
$$

where the knock-down factor $C$ is given by

$$
C=23.8\left(\frac{\sin \omega}{\bar{a}+\cot \omega / 2 \bar{t}}\right)^{0.66} .
$$

Thus, the elastic buckling stress of the egg-box is given by

$$
\frac{\sigma_{\mathrm{c}}}{Y}=C \frac{\pi}{2 \varepsilon_{\mathrm{Y}} \sqrt{3\left(1-v^{2}\right)}} \frac{\sin ^{2} \omega}{(\bar{a}+\cot \omega / 2 \bar{t})^{2}},
$$

where $\varepsilon_{\mathrm{Y}}$ is the yield strain of the solid material of the egg-box.

\subsubsection{Construction of a collapse mechanism map}

It is assumed that the egg-box material collapses by elastic buckling if the buckling stress $\sigma_{\mathrm{c}}$ is less than the densification stress $\sigma_{\mathrm{D}}$ as predicted by the travelling plastic hinge model. This can be shown graphically by plotting the collapse stress on a diagram with non-dimensional axes $\omega$ and $\bar{\rho}$ for selected values of $\bar{a}=a / t$. An example of such a collapse mechanism map is given in Fig. 8 for $\bar{a}=4$ and for the solid material properties $v=1 / 3$ and $\varepsilon_{Y}=0.007$. Tearing is assumed to occur if the longitudinal bending strain $\varepsilon_{\mathrm{L}}$ in the knuckle (11) exceeds the ductility of the solid material $\varepsilon_{\mathrm{f}}$. This strain is a maximum in the early stages of deformation and we assume that the material will tear if $\varepsilon_{\mathrm{L}} \geqslant \varepsilon_{\mathrm{f}}$ at $u=0$. Boundaries truncating the travelling hinge regime for $\varepsilon_{\mathrm{f}}=0.3$ and 0.5 are included in the collapse mechanism map.

In selecting materials for an energy absorption application it is common to maximise the energy absorption per unit mass $\bar{W}_{\mathrm{m}}$ at a prescribed safe level of stress $\bar{\sigma}$. To gauge the capacity of the egg-box material to absorb energy at a given stress level we have added to Fig. 8 contours of the normalised energy per unit mass $\bar{W}_{\mathrm{m}}$

$$
\bar{W}_{\mathrm{m}}=\frac{W_{\mathrm{m}} \rho_{\mathrm{s}}}{Y}
$$

and contours of normalised densification stress $\bar{\sigma}=\sigma_{\mathrm{D}} / Y$. Both $\bar{W}_{\mathrm{m}}$ and $\bar{\sigma}$ increase along the leading diagonal of the map. We note that at a given stress level $\bar{\sigma}$, the maximum energy absorption $\bar{W}_{\mathrm{m}}$ is achieved along the boundary between the travelling hinge and tearing regimes. 


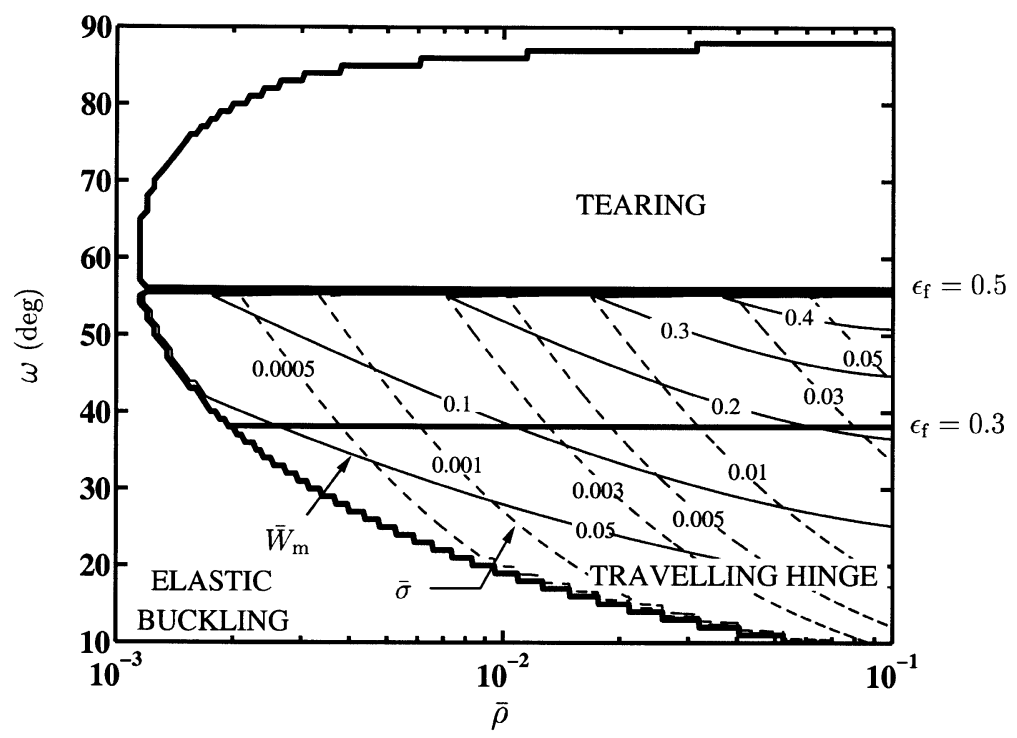

Fig. 8. Deformation mechanism map for egg-box material with $\bar{a}=4\left(\varepsilon_{\mathrm{Y}}=7 \times 10^{-3}\right.$ and $\left.v=1 / 3\right)$. Contours of the normalised densification stress $\bar{\sigma}$ and the normalised energy absorption per unit mass $\bar{W}_{\mathrm{m}}$ are included in the travelling plastic hinge deformation regime.

Fig. 9 shows the collapse mechanism map for $\bar{a}=10$, with lines truncating the travelling hinge regime due to tearing of the solid material for the choices $\varepsilon_{\mathrm{f}}=0.2$, 0.3 and 0.5. Again it is evident that at any given stress level $\bar{\sigma}$, the maximum energy absorption $\bar{W}_{\mathrm{m}}$ is achieved along the boundary between the travelling hinge and tearing regimes.

\subsubsection{Minimum weight design}

The maximum energy absorption per unit mass $\bar{W}_{\mathrm{m}}^{\max }$ is plotted in Fig. 10 as a function of $\bar{\sigma}$, for the selected values $\bar{a}=4$ and 10 , with $\varepsilon_{Y}=0.007, v=1 / 3$ and $\varepsilon_{\mathrm{f}}=0.3$. These curves correspond to boundaries of the travelling hinge and tearing regimes of Figs. 8 and 9. Fig. 10 includes estimates of $\bar{W}_{\mathrm{m}}$ for metal foams and metal honeycombs loaded in the out-of-plane direction. We assume that the compressive strength of the metal foam is given by Ashby et al. (2000)

$$
\frac{\sigma_{\text {foam }}}{Y}=0.3 \bar{\rho}^{1.5}
$$

where $Y$ is the yield strength of the fully dense metal and $\bar{\rho}$ the relative density of the foam. Employing the same notation, the average axial collapse stress for hexagonal honeycombs is given by Gibson and Ashby (1997)

$$
\frac{\sigma_{\mathrm{hc}}}{Y} \approx 4.85 \bar{\rho}^{5 / 3}
$$




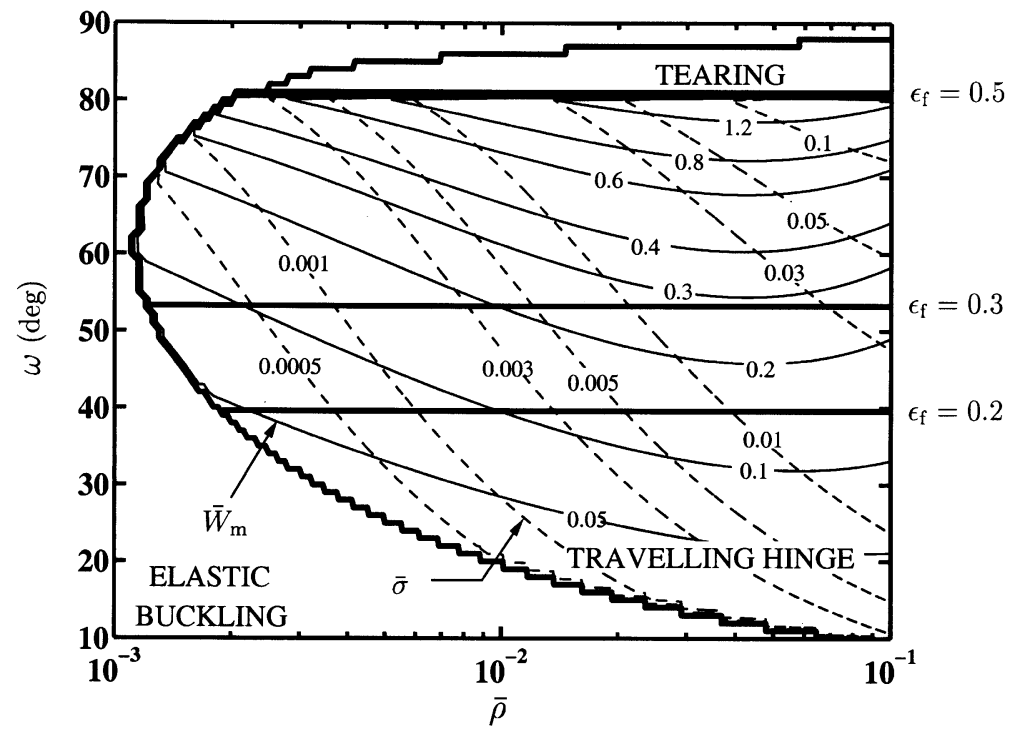

Fig. 9. Deformation mechanism map for egg-box material with $\bar{a}=10\left(\varepsilon_{\mathrm{Y}}=7 \times 10^{-3}\right.$ and $\left.v=1 / 3\right)$. Contours of the normalised densification stress $\bar{\sigma}$ and the normalised energy absorption per unit mass $\bar{W}_{\mathrm{m}}$ are included in the travelling plastic hinge deformation regime.

The densification strain $\varepsilon_{\mathrm{D}}$ for these materials is taken as (Ashby et al., 2000)

$$
\varepsilon_{\mathrm{D}}=0.8-1.75 \bar{\rho} .
$$

Upon making use of these relations, the normalised energy absorption per unit mass $\bar{W}_{\mathrm{m}}$ is plotted in Fig. 10 as a function of the normalised collapse strength $\bar{\sigma}$. We note that the egg-box material substantially out-performs metal foams and compares favourably with hexagonal honeycombs from an energy absorption standpoint for $\bar{\sigma}<0.01$.

\subsection{Practical design considerations}

The optimisation discussed above indicates that egg-box materials with large cone angles $\omega>60^{\circ}$ (and thus correspondingly high values of $\bar{a}$ to avoid tearing) are highly efficient energy absorbers. However, manufacturing constraints may restrict the magnitude of the cone angle $\omega$ : the sheet may tear during pressing to the egg-box shape at too large a value of $\omega$.

It is instructive to explore the dependence of the energy absorption for an egg-box material upon the value of $\bar{a}$, at a fixed cone angle $\omega$. Curves of $\bar{W}_{\mathrm{m}}$ as a function of the normalised stress $\bar{\sigma}$ are shown in Fig. 11 for a cone angle $\omega=30^{\circ}$, with $\bar{a}=4$ and 10. Note that for an assumed material failure strain $\varepsilon_{\mathrm{f}}=0.3$, these geometries lie within the travelling hinge regime of behaviour. The corresponding optimal energy absorption designs for $\bar{a}=4\left(\omega=38^{\circ}\right)$ and $\bar{a}=10\left(\omega=52^{\circ}\right)$ which lie on the boundary of the travelling hinge and tearing regimes and are included in Fig. 11 for comparison 


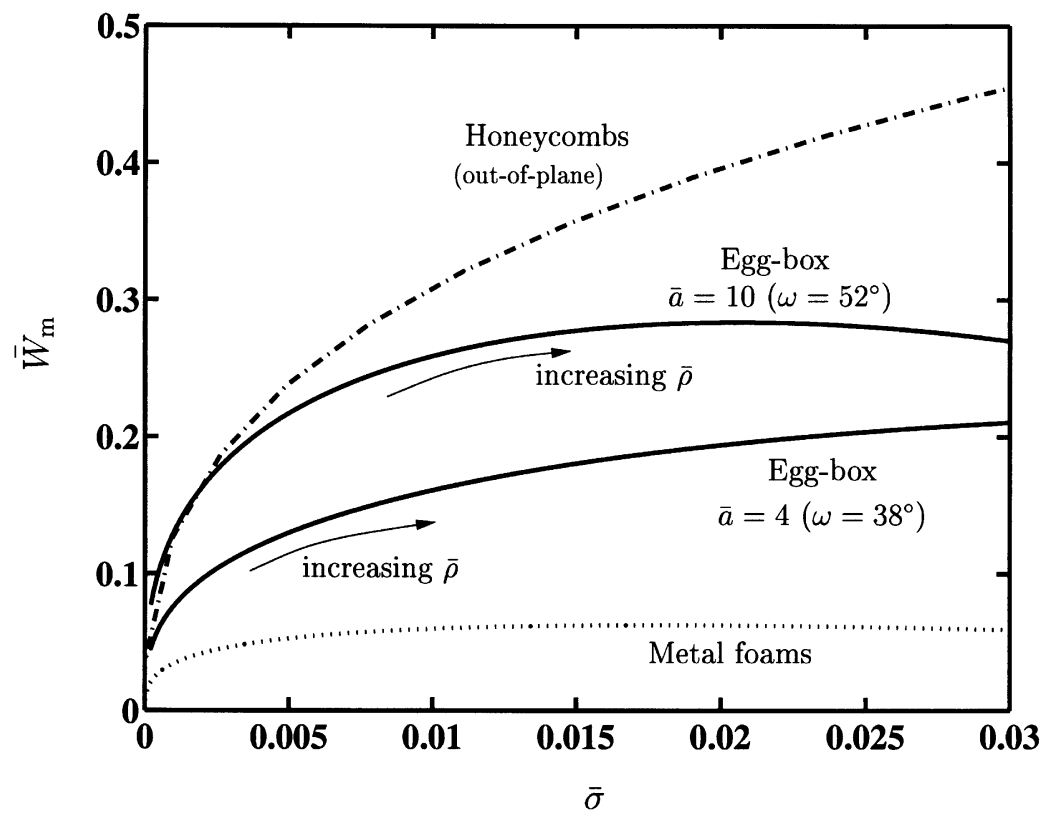

Fig. 10. Energy absorption per unit mass versus the normalised stress of the optimised egg-box material for egg-box materials with $\bar{a}=4$ and $10\left(\varepsilon_{\mathrm{Y}}=0.007, v=1 / 3\right.$ and $\left.\varepsilon_{\mathrm{f}}=0.3\right)$. For comparison, curves for metallic foams and out-of-plane crushing of hexagonal honeycombs are included.

purposes. We see from Fig. 11 that, for a given value of angle $\omega$, the lower value of $\bar{a}$ provides the more efficient energy absorber. In fact, $\bar{W}_{\mathrm{m}}$ is maximised by selecting the minimum value of $\bar{a}$ without tearing (for the given value of cone angle $\omega$ ). This optimal choice of $\bar{a}$, designated $\bar{a}_{\text {min }}$ (and defined via Eq. (11) with $u=0$ and $\varepsilon_{\mathrm{L}}=\varepsilon_{\mathrm{f}}$ ) is plotted in Fig. 12 as a function of $\omega$ for selected values of the ductility $\varepsilon_{\mathrm{f}}$. This diagram may be interpreted as follows: tearing is avoided by choosing egg-box geometries to the left of the line for a given ductility $\varepsilon_{\mathrm{f}}$, with the optimal design along the line. Thus, Fig. 12 may be used as a practical design chart to select the value of $\bar{a}$, assuming that $\omega$ has already been fixed by manufacturing constraints and the material failure strain $\varepsilon_{\mathrm{f}}$ is known.

\section{Discussion}

The above analysis demonstrates that the energy absorption capacity of egg-box material compares favourably with competing materials such as metal foams and honeycombs. Unlike metal foams which deform by stationary plastic hinges within the cellular micro-structure, the egg-box material deforms by travelling plastic hinges which sweep through the micro-structure. Additionally, for the egg-box material, each material element that enters and leaves the travelling hinge undergoes cyclic plastic straining. 


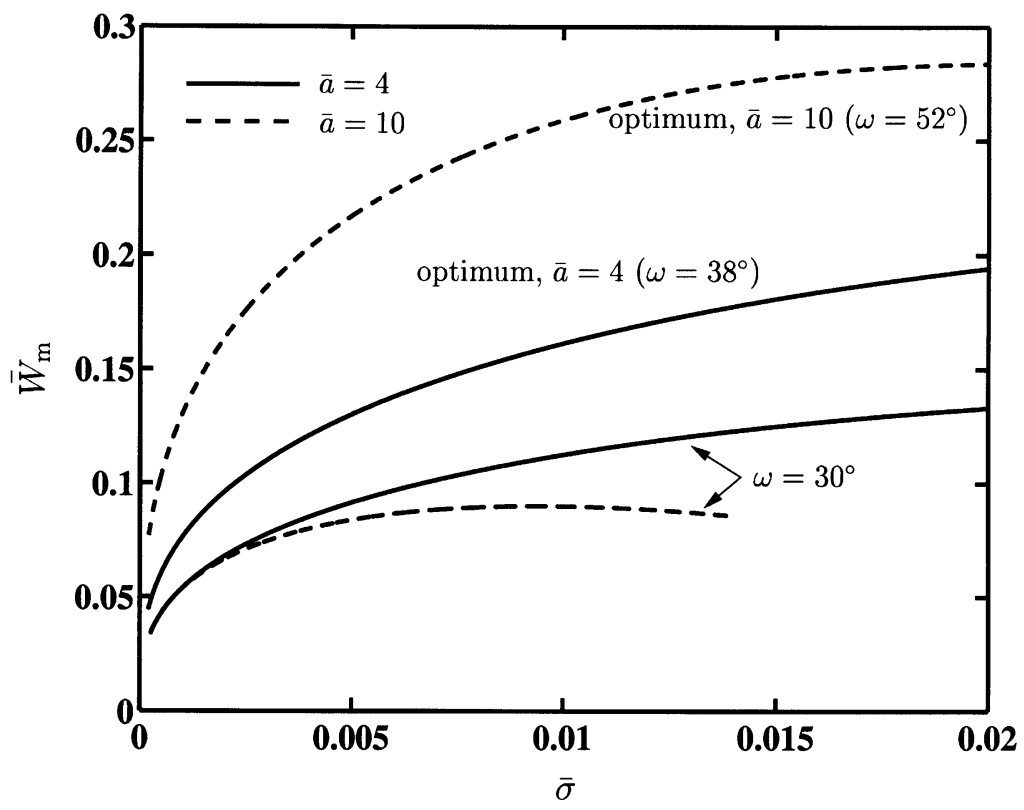

Fig. 11. The energy absorption for $\bar{a}=4,10$ and $\omega=30^{\circ}$ is shown to demonstrate that for any given $\omega$ in the travelling hinge regime, a lower $\bar{a}$ gives a higher $\bar{W}_{\mathrm{m}}$. For comparison, the optimum energy absorption from Fig. 10 is included for $\bar{a}=4$ and 10 .

This mechanism of reversed plasticity is also observed in the inversion of tubes (Reid, 1993 ).

The optimisation conducted in this study has revealed that the ideal egg-box geometry for energy absorption at a given stress level has the following geometric features:

1. a large cone angle $\omega$, provided the egg-box can be manufactured without tearing,

2. a flat top which is of sufficiently large radius $\bar{a} \equiv a / t$ for collapse to occur without tearing, and

3. a transition between the flat top and the conical portion of radius $c / t \approx 2$ in order to establish the travelling hinge collapse mode without a load peak, (see Fig. 5b).

In the experiments described above tearing was observed for frustra with large cone angles $\omega$ and small $\bar{a}$. This resulted in collapse by a plastic folding mechanism. In contrast, exploratory experiments on thick-walled conical frustra showed that tearing can be accompanied by a travelling plastic hinge. This petalling phenomenon has been observed previously in the inversion of tubes, see Reddy and Reid (1986). An analysis of this phenomena by Atkins (1987) suggests that the number of petals is a function of the tube diameter, the strength to toughness ratio of the metal and the hoop fracture 


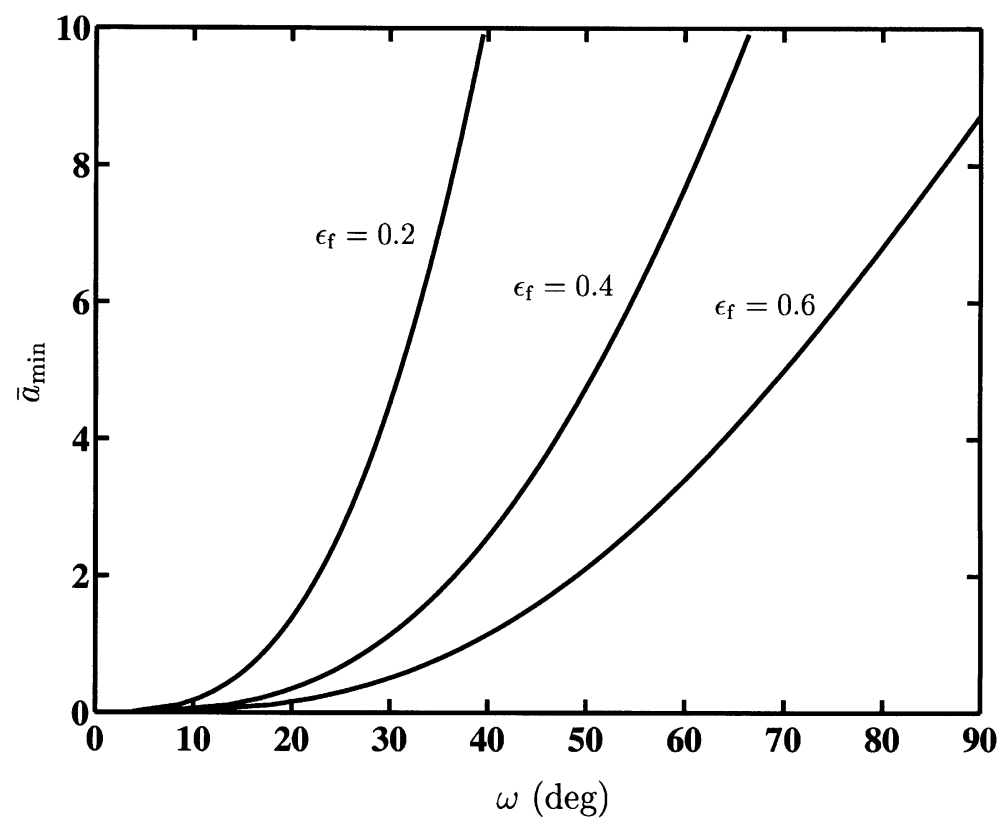

Fig. 12. Minimum value of $\bar{a}$ to avoid tearing for a given cone angle $\omega$ at selected values of ductility $\varepsilon_{\mathrm{f}}$.

strain. An analysis of the tearing of conical shells under axial compression remains a topic for future study.

\section{Concluding remarks}

A set of experiments on the axial compression of closed-top conical frustra revealed that collapse was by a travelling hinge mechanism. If the induced strain within the knuckle exceeded the ductility of the material, tearing occurred and the collapse mechanism switched to plastic folding, as observed previously by Mamalis et al. (1986a, b) for open-top conical frustra.

An egg-box material comprising a square array of conical frustra was manufactured by cold-pressing sheet aluminium. The egg-boxes were subjected to transverse compression, with constraint against in-plane macroscopic straining. In all cases, collapse was by a travelling plastic hinge within each unit cell of the egg-box. An analytical model of the travelling hinge mode has been derived, and a collapse mechanism map constructed. This map displays the competing collapse modes of elastic buckling, travelling plastic hinge and material tearing. The egg-box geometry was optimised to maximise the energy absorption per unit mass at a given stress level: the optimum design lies along the boundary between the travelling hinge and material tearing regimes of the collapse mechanism map. The optimised egg-box material compares very favourably with the competing concepts of metal foams and honeycombs for energy absorption. 


\section{Acknowledgements}

The authors are grateful to DARPA/ONR for their financial support through MURI grant number N00014-1-96-1028 on the Ultralight Metal Structures project at Harvard University. The authors would like to thank Prof. M.F. Ashby for helpful discussions, and S. Marshall and A. Heaver for help with the experiments.

\section{References}

Alghamdi, A.A.A., 2001. Collapsible impact energy absorbers: an overview. Thin-walled Struct. 39, $189-213$.

Ashby, M.F., Evans, A.G., Fleck, N.A., Gibson, L.J., Hutchinson, J.W., Wadley, H.N.G., 2000. Metal Foams: A Design Guide. Butterworth-Heinemann, Stoneham, MA.

Ashmead, M., 2000. Energy-absorbing structures. International Patent, Serial No. WO 00/31434, June 2000. Atkins, A.G., 1987. On the number of cracks in the axial splitting of ductile metal tubes. Int. J. Mech. Sci. 29 (2), 115-121.

Banhart, J., 2000. Manufacturing routes for metallic foams. J. Miner. Mater. Soc. 52 (12), $22-27$.

Calladine, C.R., 1986. Analysis of large plastic deformations in shell structures. In: Bevilacqua, L., Feijoo, R., Valid, R. (Eds.), Inelastic Behaviour of Plates and Shells, IUTAM Symposium. Springer, Rio de Janerio, pp. 69-101.

Deshpande, V.S., Fleck, N.A., 2001. Collapse of truss core sandwich beams in 3-point bending. Int. J. Solids Struct. $38(36,37), 6275-6305$.

Deshpande, V.S, Fleck, N.A., Ashby, M.F., 2001. Effective properties of the octet-truss lattice material. J. Mech. Phys. Solids 49 (8), 1747-1769.

Gibson, L.J., Ashby, M.F., 1997. Cellular Solids: Structure and Properties, 2nd Edition. Cambridge University Press, Cambridge.

HKS, 1997. ABAQUS/Standard Users Manual, Version 5.7. Hibbit, Karlsson and Sorensen Inc., Providence, RI.

Mamalis, A.G., Manolakos, D.E., Saigal, S., Viegelahn, G.I., Johnson, W., 1986a. Extensional plastic collapse of thin-wall frustra as energy absorbers. Int. J. Mech. Sci. 28 (4), 219-229.

Mamalis, A.G., Manolakos, D.E., Viegelahn, G.I., Vaxevanidis, N.M., Johnson, W., 1986b. On the inextensional axial collapse of thin PVC conical shells. Int. J. Mech. Sci. 28 (5), 323-335.

Onat, E.T., 1955. The plastic collapse of cylindrical shells under axially symmetric loading. Quart. Appl. Math. 13, 63-72.

Reddy, T.Y., Reid, S.R., 1986. Axial splitting of circular metal tubes. Int. J. Mech. Sci. 28 (2), 111-131.

Reid, S.R., 1993. Plastic deformation mechanisms in axially compressed metal tubes used as energy absorbers. Int. J. Mech. Sci. 35 (12), 1035-1052.

Seide, P., 1956. Axisymmetrical buckling of circular cones under axial compression. J. Appl. Mech. 23, 625-628.

Wicks, N., Hutchinson, J.W., 2001. Optimal truss plates. Int. J. Solids Struct. 38 (30,31), 5165-5183. 